\title{
An Adaptive and Time-Efficient ECG R-Peak Detection Algorithm
}

\author{
Qin Qin, Jianqing Li, Yinggao Yue, and Chengyu Liu \\ School of Instrument Science and Engineering, Southeast University, Nanjing 210018, China \\ Correspondence should be addressed to Jianqing Li; ljq@seu.edu.cn
}

Received 16 March 2017; Revised 19 June 2017; Accepted 12 July 2017; Published 6 September 2017

Academic Editor: Ioannis G. Tollis

Copyright ( 2017 Qin Qin et al. This is an open access article distributed under the Creative Commons Attribution License, which permits unrestricted use, distribution, and reproduction in any medium, provided the original work is properly cited.

R-peak detection is crucial in electrocardiogram (ECG) signal analysis. This study proposed an adaptive and time-efficient R-peak detection algorithm for ECG processing. First, wavelet multiresolution analysis was applied to enhance the ECG signal representation. Then, ECG was mirrored to convert large negative R-peaks to positive ones. After that, local maximums were calculated by the first-order forward differential approach and were truncated by the amplitude and time interval thresholds to locate the R-peaks. The algorithm performances, including detection accuracy and time consumption, were tested on the MIT$\mathrm{BIH}$ arrhythmia database and the QT database. Experimental results showed that the proposed algorithm achieved mean sensitivity of $99.39 \%$, positive predictivity of $99.49 \%$, and accuracy of $98.89 \%$ on the MIT-BIH arrhythmia database and $99.83 \%$, 99.90\%, and 99.73\%, respectively, on the QT database. By processing one ECG record, the mean time consumptions were $0.872 \mathrm{~s}$ and $0.763 \mathrm{~s}$ for the MIT-BIH arrhythmia database and QT database, respectively, yielding $30.6 \%$ and $32.9 \%$ of time reduction compared to the traditional Pan-Tompkins method.

\section{Introduction}

Electrocardiogram (ECG) can describe the electrical activity of the heart and is an essential tool for the diagnosis of cardiovascular diseases (CAD). With the rapid development of wearable and wireless ECG techniques, real-time and routine ECG monitoring is attracting more and more attention due to the increasing popularization of medical health, especially for the elderly people [1]. Recent years, lots of publications about ambulatory ECG monitoring devices have been reported [2-4], aiming to automatically monitor the heart activities and give the feedback of any CAD early warning in real time. However, this application still needs significant development due to the challenge of unexpected noise effects in ECG signal, such as baseline drift, electrode motion and stretching, motion artifacts, and muscle noise $[5,6]$, which impedes the automatic ECG processing technology to perform effectively. The primary sources of noises are electrical activities of muscles and baseline drift caused by respiration, poor contact of electrodes, and equipment or electronic devices $[7,8]$. Electrode movement alters the signal baseline and brings the signal fluctuate perpendicularly to the baseline. If the electrode moves drastically enough to drop from the skin, baseline drift will overwhelm the signal and waveform distortion occurs [9]. Motion artifact is generally attributed to the variation of electrode-skin impedance during a subject's motion. Changed impedance will be treated by the ECG amplifier as a different input, resulting in impedance mismatching and difficult identification of irregular fluctuation on small amplitude waveforms, such as $\mathrm{P}$ wave and $\mathrm{T}$ wave $[10,11]$. Consequently, noise removal is the preliminary issue to consider for in ECG signal processing.

ECG features are essential characteristics for CAD diagnosis. R-peak detection is the datum since all other features are extracted after the R-peak location [12]. Accurate Rpeak detection is critical for arrhythmia diagnosis such as atrial premature contraction, tachycardia, and bradycardia [13]. Nevertheless, efficient R-peak extraction is still difficult in the dynamic and noisy environment due to the timevarying waveform morphology. This would be more difficult when ECG signal is overwhelmed by noises with similar frequency in energy distribution [9].

Over the last decades, numerous techniques have been proposed for R-peak detection. In [14], a thorough review 
on R-peak detection methodologies for portable, wearable, battery-operated, and wireless ECG devices was elaborated. The authors claimed that the thresholding methods were regarded as the most computationally efficient. However, the suitable parameter settings for thresholds were difficult. The most widely used R-peak detection method, proposed by Pan and Tompkins [15], is the Pan-Tomkins method. It is a threshold-based method with low complexity. Other algorithms of R-peak detection can be classified as pattern recognition [16, 17], wavelet transform [18], mathematical morphology [19], and digital filter [20]. In [10], a real-time R-peak detector using adaptive thresholding was proposed. This algorithm consisted of preprocessing to initialize Rpeak threshold and thresholding to adaptively modify the threshold. It achieved sensitivity and positive predictivity higher than $99.3 \%$. In [21], a different interference-based method was developed. This method could effectively distinguish R-peaks from high amplitude noises but failed to detect R-peaks when abrupt jump of baseline appeared. Some researchers also conducted ECG feature extraction without predenoising $[22,23]$. The detection accuracy could reach up to $94.8 \%$, although much lower than that acquired from the denoised signals.

Time cost is important due to the fast-responding requirement in $\mathrm{CAD}$ early warning applications [4], especially in the real-time monitoring. Many ambulatory ECG devices are generally limited in power supply and computation [1]. The conventional feature extraction algorithms are, from a computational perspective, very intensive tasks, which are typically executed in mainframe-type computational facilities. A significant power expenditure component in such systems is the energy required by the radio front-end for supporting continuous data transmission, which may not allow a long-term sustainable operation. To this end, some researchers have attempted to develop algorithms of low computational load. Apart from the aforementioned methods, in [24], the authors presented a low-complexity ECG feature extraction approach for mobile healthcare applications. This technique was based on the combination of wavelet analysis and time-domain morphology principles. Except for high accuracy and precision, low computation and fast response are also needed in ECG feature extraction.

In this study, an adaptive and time-efficient ECG R-peak detection algorithm is proposed. The method takes advantage of wavelet-based multiresolution analysis (WMRA) and adaptive thresholding. WMRA is applied to strengthen ECG signal representation by extracting ECG frequency interval of interest from wide-range frequencies, which contain interference such as baseline drift and motion artifacts. All the noises produce considerable influence on the following thresholding operation. The adaptive thresholding is designed to exclude false $\mathrm{R}$-peaks in the reconstructed signal by WMRA. The proposed algorithm was tested by the MIT-BIH arrhythmia database (MITDB) and the QT database (QTDB) [25]. Both accuracy and time consumption of the algorithm were evaluated. By exploring the timefrequency property of ECG, this study aims to conduct preliminary and tentative research on adaptive and time- efficient R-peak detection for low-quality ECG signals, promoting automatic ECG processing technology for clinical and daily use.

The remainder of the paper is organized as follows. Section 2 elaborated the detailed procedures of the proposed R-peak detection algorithm. In Section 3, experiment setups were introduced, including the datasets and the evaluation indices. Section 4 demonstrated the experimental results over R-peak detection accuracy, time consumption and time complexity, and the selection of optimal threshold coefficients. Section 5 discussed the advantages and the potential limitation of our algorithm. The summarization of this study was presented in Section 6.

\section{Proposed R-Peak Detection Algorithm}

The R-peak detection system is described in Figure 1. The purpose of this study is to develop an algorithm which can effectively identify R-peaks mixed in different noises.

2.1. Step 1: WMRA Enhancement. WMRA enhances signals using wavelet transform to extract both time and frequency domain information. This method is very suitable for ECG processing since ECG is essentially nonstationary with small amplitude $(0.01 \sim 5 \mathrm{mV})$ and low frequency $(0.05 \sim 100 \mathrm{~Hz})$ [26]. This method also provides low computational cost [27]. By WMRA, signal below $0.05 \mathrm{~Hz}$ and above $100 \mathrm{~Hz}$ can be filtered from the raw signal. These intervals are not the ECG frequency bands and contain most types of noises [28]. In addition, according to the Nyquist criterion, subfrequency band presented by each decomposition level is directly related to the sampling frequency $f_{s}$ [26]. Consequently, the ECG signals, sampled at $360 \mathrm{~Hz}$ in MITDB and $250 \mathrm{~Hz}$ in QTDB as illustrated in [25], are all decomposed up to 8 levels in this study.

Figure 2 shows the decomposition procedure of eightlevel WMRA by using bior6.8 wavelet. For MITDB, $\mathrm{CD}_{2} \sim \mathrm{CD}_{8}$ consist of frequency components in a range of $0.70-90 \mathrm{~Hz}$, which is the ECG frequency band of interest. $\mathrm{cD}_{1}$ with frequency band $90 \sim 180 \mathrm{~Hz}$ and $\mathrm{cA}_{8}$ with frequency band $0 \sim 0.70 \mathrm{~Hz}$ are beyond the ECG frequency; they are not the considered coefficients containing baseline drift and other interference. Consequently, $\mathrm{cD}_{1}$ and $\mathrm{CA}_{8}$ are set to zeroes; $\mathrm{CD}_{2} \sim \mathrm{CD}_{8}$ are kept for reconstruction. Similarly, for QTDB, $\mathrm{cA}_{8}$ with frequency band $0 \sim 0.49 \mathrm{~Hz}$ is set to zero; $\mathrm{CD}_{1} \sim \mathrm{CD}_{8}$ with frequency components in a range of $0.49-$ $125 \mathrm{~Hz}$ are kept. All the retained coefficients are then filtered by the wavelet shrinking threshold algorithm [29]. In this study, soft thresholding is adopted due to its good continuity and no Gibbs phenomenon on step points [30].

2.2. Step 2: Signal Mirroring. For some ECG patterns, such as premature ventricular contraction (PVC) beat, R-peaks are presented with amplitude below the baseline but other features are above the baseline. To avoid the potential missing detection, signal mirroring is designed. The mirroring procedure for a PVC segment is described in Figure 3. Large negative amplitudes are mirrored by taking the baseline as their symmetrical axis. However, not all the negative 


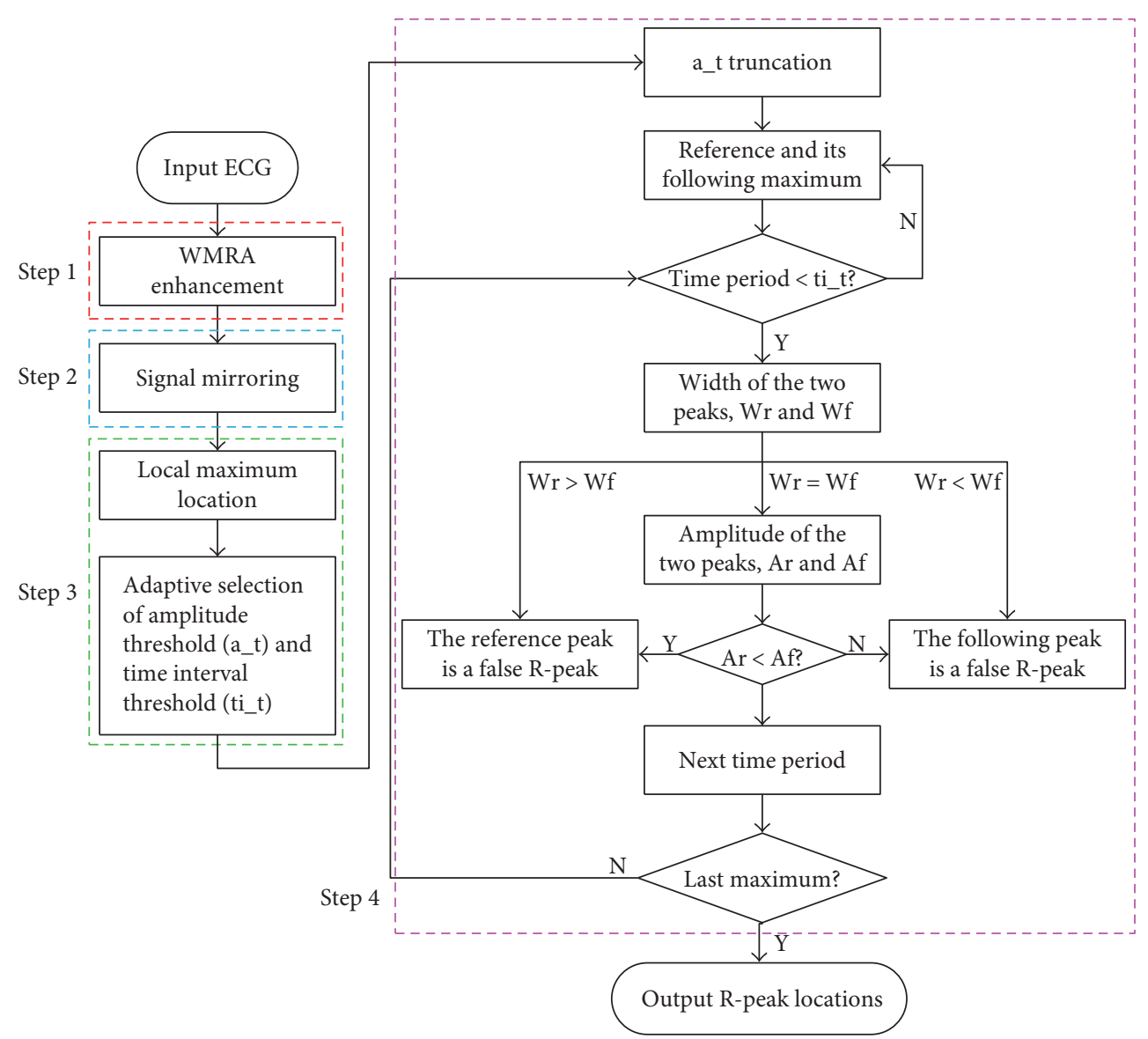

FIGURE 1: Block diagram of the proposed R-peak detection algorithm.

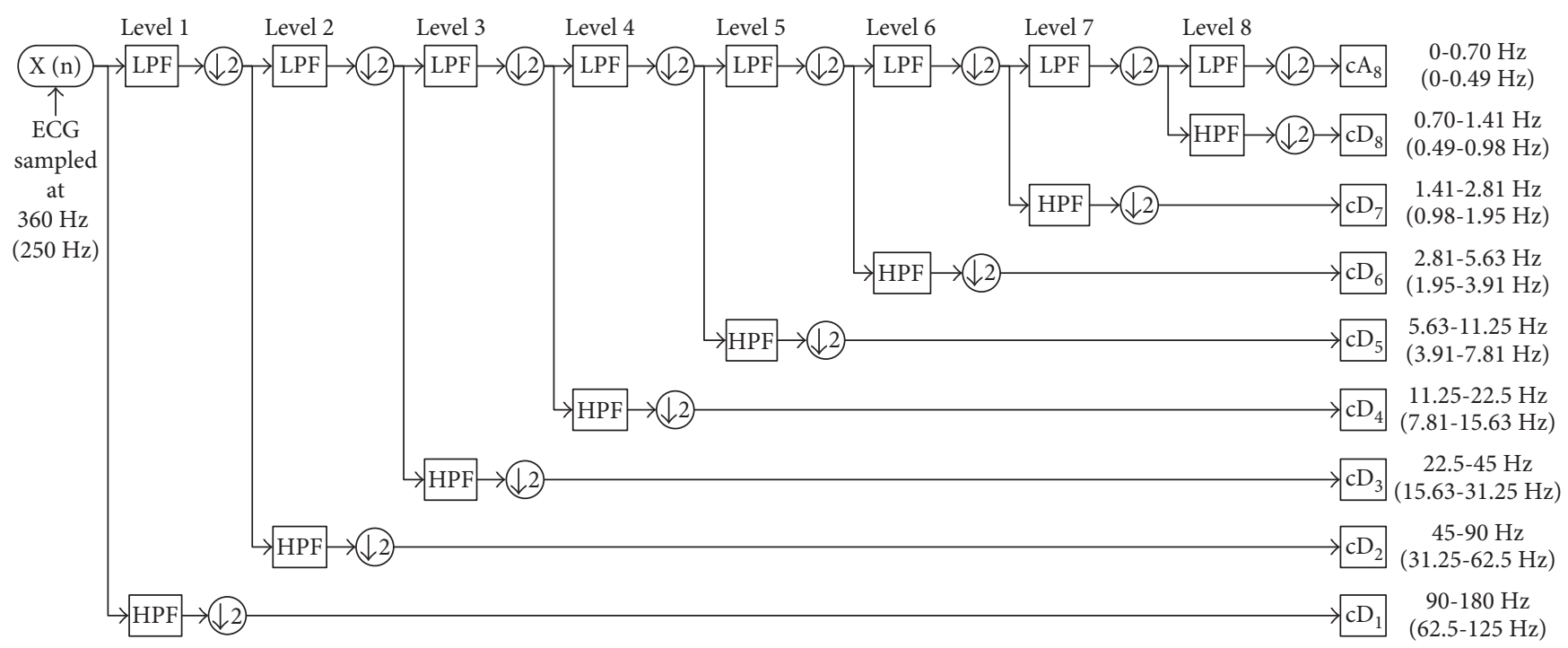

LPF: Low-pass filter HPF: High-pass filter

Figure 2: Decomposition process of the eight-level WMRA. The sampling frequency is decomposed into two subbands: high frequency of detail coefficient $\left(\mathrm{cD}_{j}\right)$ and low frequency of approximation coefficient $\left(\mathrm{cA}_{j}\right)$, both in Level $j$. 
amplitudes are mirrored, they should be significantly distinctive from adjacent negative values. This assumption is based on the fact that R-peaks have steep slopes while other waves such as $\mathrm{P}$ wave and $\mathrm{T}$ wave have gentle ones [10]. Steep slope means drastic increment and decrement on both sides of local maximum, and the slope is finished within several sampling points. If the absolute amplitude of a negative point is 1.5 times larger than that of the adjacent points within 0.278 seconds $\left(0.278 f_{\text {s }}\right.$ points $)$ before and after it, then the negative point will be mirrored.

$$
\text { Negative }\left\{\begin{array}{cl}
\text { mirrored } & \text { if } \forall \max \left\{0, k-0.278 f_{\mathrm{s}}\right\} \leq i \leq \min \left\{L, k+0.278 f_{\mathrm{s}}\right\}, i \neq k, \exists\left|A_{N}[k]\right| \geq 1.5\left|A_{A}[i]\right| \\
\text { unchanged } & \text { else, }
\end{array}\right.
$$

where $L$ is the signal length, $A_{N}[k]$ is the amplitude of large negative point with position number $k$ in signal, $0<k \leq L$, and $A_{A}[i]$ is the amplitude of point within $0.278 \mathrm{~s}$ before and after the large negative point.

In some literatures $[15,21,31-33]$, authors recommend that signal with baseline drift removed could be squared to highlight the difference between true R-peaks and false ones, such as high-amplitude noise and high-amplitude $\mathrm{P}$ waves. However, this operation may not be suitable in our method due to the differences among R-peak amplitudes. If the signal is squared, amplitude values below 1 will be smaller than the original, and values above 1 will be enlarged. This increases the difference among true R-peaks and is adverse for the amplitude threshold to detect potential R-peaks, especially when a signal segment is mixed with large and small amplitude R-peaks.

2.3. Step 3: Local Maximum Location and Adaptive Threshold Selection. Local maximums are located by implementing first-order forward differential in the mirrored signal. The procedure is illustrated as follows.

(1) First-order forward difference is implemented on ECG signal with $\Delta \mathrm{ECG}[n]=\operatorname{ECG}[n+1]-\operatorname{ECG}[n]$.

(2) For all the elements in $\triangle \operatorname{ECG}[n]$, values less than, equal, and more than 0 are replaced by $-1,0$, and 1 , respectively,

$$
\Delta \mathrm{ECG}[n] \leftarrow \operatorname{sgn}(\Delta \mathrm{ECG}[n])= \begin{cases}1 & \Delta \mathrm{ECG}[n]>0 \\ 0 & \Delta \mathrm{ECG}[n]=0 \\ -1 & \Delta \mathrm{ECG}[n]<0 .\end{cases}
$$

(3) First-order forward difference is implemented on the updated $\triangle \mathrm{ECG}[n]$, and the value of $\triangle \mathrm{ECG}[n]$ is symbolized by $-2,0$, and 2 . Local maximums in original ECG signal are positions shifted by 1 sample to the right of -2 .

The following threshold procedure depends significantly on the amplitude threshold ( $\mathrm{a}_{-} \mathrm{t}$ ) and time interval threshold (ti_t), which are adaptively determined by the location of local maximum instead of adopting fixed thresholds, since fixed thresholds do not copy with large or small amplitude $\mathrm{R}$-peak and slow or fast beat. The selection of the two thresholds is displayed in Figure 4. During an ECG segment, a_t is selected to be a multiple of the amplitude maximum $\mathrm{MAX}_{\text {seg_amp }}$; ti_t is selected to be a multiple of the average horizontal distance of each adjacent local maximum $\mathrm{AVE}_{\text {max_dis. }}$. If the positions and amplitudes of these local maximums change, the two thresholds will change correspondingly; hence, a_t and ti_t will adjust adaptively according to the maximum variety.

$$
\left\{\begin{array}{l}
\mathrm{a} \_t_{-}=K_{\text {amp }} \mathrm{MAX}_{\text {seg_amp }} \\
\mathrm{ti \_ t}=K_{\text {time }} \mathrm{AVE}_{\text {max_dis }} .
\end{array}\right.
$$

However, the threshold selection is strongly dependent on the noise; $K_{\mathrm{amp}}$ and $K_{\text {time }}$ are coefficients designed to correct the noise influence. The detailed selection method for them is discussed in Section 4.3. In a segment, the positions of local maximums are fixed, correspondingly; MAX $_{\text {seg_amp }}$ and $\mathrm{AVE}_{\text {max_dis }}$ are deterministic. Hence, only $K_{\mathrm{amp}}$ and $K_{\text {time }}$ need to be decided. The thresholds are automatically updated by the shift of new coming segment. The superiority of automatic threshold substitution embodies in the corresponding adjustment on recognition for small amplitude and slow or fast cardiac beat, as fixed thresholds may fail to detect R-peaks in these cases.

2.4. Step 4: Threshold Recognition. Actually, most of the local maximums are not true R-peaks, such as burst points caused by high-frequency interference. The difficulty of R-peak detection lies in the recognition of false R-peaks with amplitudes approximate to or even larger than true R-peaks. To this end, a_t is designed to filter the local maximums with small amplitudes. In general, there should be no extra Rpeaks between two adjacent R-peaks; otherwise, the extra $\mathrm{R}$-peaks are definitely false. Assisted by this knowledge, ti_t is designed to further remove false R-peaks omitted by a_t. The thresholding algorithm is plotted in Figure 5. The example ECG is from the Record 200 in MITDB with PVC beats. It comprises of large negative R-peaks, and consequently, the signal needs to be mirrored. The marks $M$ in Figure 5 signify the mirrored R-peaks, where they should originally be large negative amplitudes. After the amplitude filtration, the time interval threshold algorithm is summarized as follows:

(1) Step A. A local maximum and its following maximum are chosen as true reference (Tref) and comparative 


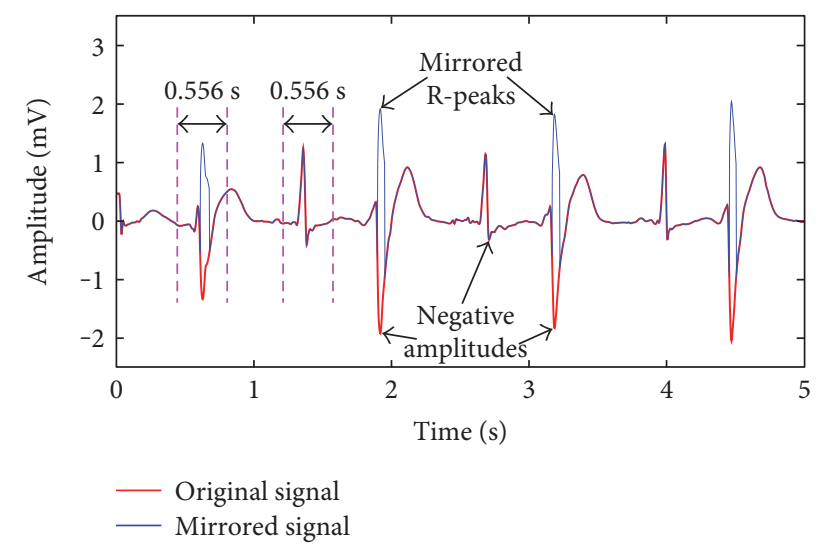

Figure 3: Signal mirroring example with comparison range of $0.556 \mathrm{~s}$ and amplitude multiple of 1.5 .

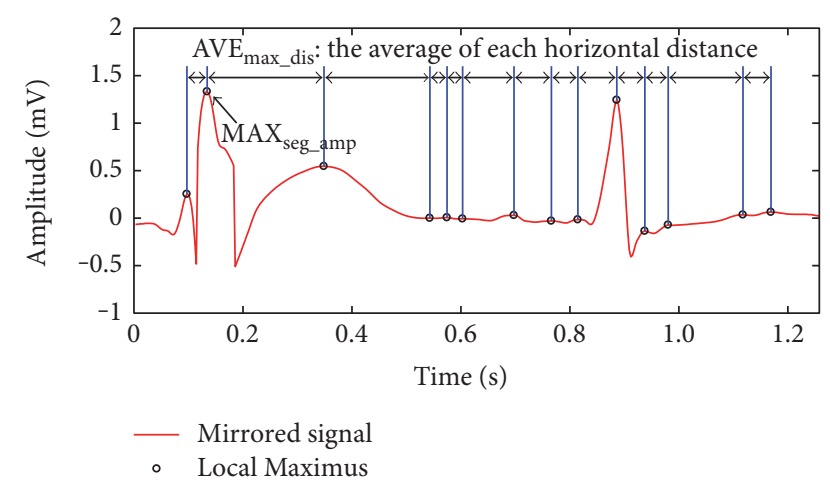

FIgURE 4: Determination of $a_{\_} t$ and ti_t by dimensionless coefficients $K_{\text {amp }}$ and $K_{\text {time }}$.

reference (Cref) respectively, turn to Step B. If there is no Cref, Tref is considered as a true R-peak and the algorithm ends.

(2) Step B. The time period ( $\left.t_{-} p\right)$ between Tref and Cref is calculated. If $t \_p<t i \_t$, it indicates that one of the two maximums is a false R-peak, then turn to Step C. Otherwise, Tref is considered as a true R-peak, Cref replaces Tref as true reference, then turn to Step A for next thresholding.

(3) Step C. Widths along the baseline of Tref $(W r)$ and Cref $(W f)$ are compared, if $W r<W f$, Tref is considered to be a true R-peak, if $W r>W f$, Cref is treated as a true R-peak [34]. Then turn to Step E. If $W r=W f$, turn to Step D.

(4) Step D. Amplitude of Tref (Ar) and Cref (Af) is compared, if $A r>A f$, Tref is considered to be a true Rpeak, otherwise, Cref is treated as a true R-peak [34]. Then turn to Step E.

(5) Step E. The false maximum is replaced by the third local maximum (Rref) just behind the two maximums, which is treated as a new Cref, and then return to Step B. If there is no Rref in the time period, turn to Step A.

\section{Experiment Designs}

3.1. Datasets. The MITDB comprises 48 ECG records, and each contains 30-minute ECG signal $[35,36]$, resulting in a total of 109966 beats that were all used. The ECG records have acceptable quality, sharp and tall $\mathrm{P}$ and $\mathrm{T}$ waves, negative $\mathrm{R}$ waves, small R-peak amplitudes, wider $\mathrm{R}$ waves, muscle noise, baseline drift, sudden changes in heartbeat morphology, multiform PVC, long pauses, and irregular heart rhythms [25].

The QTDB contains a total of 105 15-minute ECGs. ECGs in this database were chosen to represent a wide variety of QRS and ST-T morphologies with real-world variability to challenge the detection algorithms [35, 37]. A total of 86995 beats from 82 records were used, and the rest 23 records of sel30-sel52 were excluded since the QRS annotations were not given.

It should be noted that both databases provide two channels of ECG signals. In this study, only the first channel was used for algorithm development and test.

3.2. Evaluation Indices. Experimental results are evaluated in terms of sensitivity (SEN), positive predictivity $(+\mathrm{P})$, and accuracy (ACC). The definitions of the indices are expressed in

$$
\begin{aligned}
\mathrm{SEN} & =\frac{\mathrm{TP}}{\mathrm{TP}+\mathrm{FN}} \times 100 \%, \\
+\mathrm{P} & =\frac{\mathrm{TP}}{\mathrm{TP}+\mathrm{FP}} \times 100 \%, \\
\mathrm{ACC} & =\frac{\mathrm{TP}}{\mathrm{TP}+\mathrm{FN}+\mathrm{FP}} \times 100 \%,
\end{aligned}
$$

where TP (true positive) is the number of R-peaks correctly recognized, FN (false negative) is the number of R-peaks missed, and FP (false positive) is the number of false Rpeaks recognized as true R-peaks. The TP, FN, and FP, verified by the annotations announced in [25], are calculated based on a tolerance window of $50 \mathrm{~ms}$.

Time complexity is also tested, which quantifies the amount of time taken by an algorithm to run as a function of the length of string representing the input. It reflects the increment of time consumption when the input data increase. Time complexity of an algorithm is commonly expressed using $O$ notation. If the number of input data $n$ multiplies, the time consumption multiplies with an increment of $n^{m}$, the algorithm is called to have an $m$-order time complexity symbolized as $O\left(n^{m}\right)$. In this study, all the time cost experiments were carried out on a desktop (CPU i7-2600 3.40GHz, 8GB RAM, 64-bit Windows 7 Enterprise) installed with Matlab 2016b.

\section{Results}

First, for both databases, $K_{\text {amp }}$ and $K_{\text {time }}$ were initially set as 0.25 and 0.45 , respectively. The length of shifting signal was 
Tref = localmax 1 ; Cref = localmax 2 ; Rref=localmax $3 ; t \_p=$ time period (abs (Tref - Cref));

WTref = width (Tref); WCref = width (Cref); ATref = amplitude (Tref); ACref = amplitude (Cref);

If $t_{-} p<t i_{-} t$

If WTref $<$ WCref

localmax 1 is a true R-peak;

Else if WTref $>$ WCref

localmax2 is a true R-peak;

Else if ATref $>$ ACref

localmax1 is a true R-peak;

Else

localmax2 is a true R-peak; End if

\section{End if}

Else

localmax 1 is a true R-peak;

\section{End if}

End if

If Rref is not NULL End if

False R-peak is replaced by the Rref, and repeat the algorithm with new Tref and Cref;

Pseudocode 1: The pseudocode of the threshold procedure.

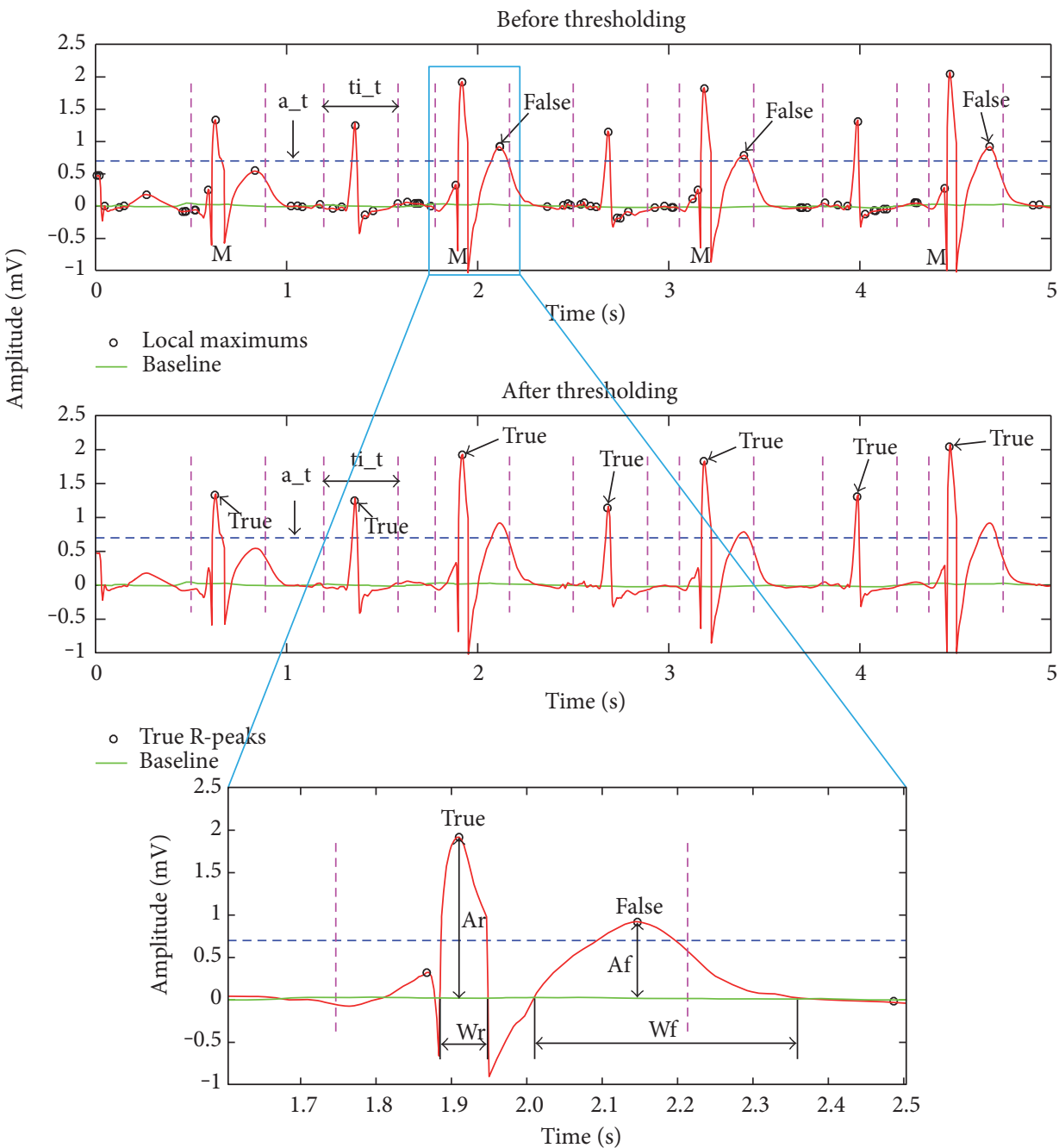

FIGURE 5: Small amplitudes filtration by a_t and false R-peak exclusion by ti_t. 
set as $10 \mathrm{~s}$ for each thresholding operation. Then, we tested the influences of the parameters $K_{\text {amp }}$ and $K_{\text {time }}$.

4.1. R-Peak Detection Results. The testing results on MITDB are summarized in Table 1 . The results demonstrate a satisfactory performance on the records. The algorithm has a total detection failure of 1229 beats (668 FN beats and $561 \mathrm{FP}$ beats) out of 109966 beats; the average SEN, +P, and ACC are $99.39 \%, 99.49 \%$, and $98.89 \%$ respectively.

The testing results on QTDB are shown in Table 2. The algorithm has a total detection failure of 238 beats (147 FN beats and 91 FP beats); out of 86995 beats, the average SEN, $+\mathrm{P}$, and ACC are $99.83 \%, 99.90 \%$, and $99.73 \%$ respectively. Compared with MITDB, the ECG signals from QTDB have much better waveforms with higher quality; distractors such as motion artifacts, burst noise, large $\mathrm{P}$, and $\mathrm{T}$ waves are much less. Consequently, the algorithm achieves a more satisfactory performance over QTDB.

Our algorithm is also compared with several existing methods, including the most widely used Pan-Tompkins method, as shown in Table 3. The comparison indicates that our algorithm achieves comparable high performance.

4.2. Time Consumption and Time Complexity. The time consumption for each record from MITDB is described in Figure 6(a). In general, the Pan-Tompkins method consumes more time than our method for most records. The mean time of this method is $1.256 \mathrm{~s}$ to process one record, while our algorithm consumes $0.872 \mathrm{~s}$, achieving about $30.6 \%$ of time reduction. Figure $6(\mathrm{~b})$ shows the time consumption ratio of the proposed method over the Pan-Tompkins method. It is obvious that our method consumes less time for most records except for records 107,109, 113, and 116, which contain large $\mathrm{T}$ waves that cause more frequent thresholding manipulations. In some cases, our algorithm economizes nearly $50 \%$ of time than the Pan-Tompkins method.

The time consumption for each record from QTDB is described in Figure 7(a) and Figure 8(a). It is obvious that our method consumes less time than the Pan-Tompkins method for all the records. The mean time of the PanTompkins method is $1.137 \mathrm{~s}$ to process one record, while our algorithm consumes $0.763 \mathrm{~s}$, achieving about $32.9 \%$ of time reduction. Figure 7 (b) and Figure 8(b) show the time consumption ratio of the proposed method over the PanTompkins method. It can be seen that all the ratios are less than 1 . The outstanding performance can be attributed to the high-quality ECG signals in QTDB.

The time consumption reveals an important characteristic of the two methods. The number of sampling points of each QTDB ECG is 225000, and the number is 650000 of each MITDB ECG. Although the number has increased about two times from QTDB to MITDB, the time consumed increases only $12.5 \%$ using our method and 9.2\% using the Pan-Tomkins method. It indicates that when data multiplies, the time consumption increases slightly instead of multiplying correspondingly. Both our method and the Pan-Tomkins method are not so sensitive to data increase.
TABLE 1: Detection results of ECG signals from MITDB.

\begin{tabular}{|c|c|c|c|c|c|c|c|}
\hline Record & $\begin{array}{l}\text { Total } \\
\text { beats }\end{array}$ & $\mathrm{TP}$ & FN & FP & SEN (\%) & $+\mathrm{P}(\%)$ & $\operatorname{ACC}(\%)$ \\
\hline 100 & 2273 & 2273 & 0 & 0 & 100.00 & 100.00 & 100.00 \\
\hline 101 & 1865 & 1864 & 1 & 1 & 99.95 & 99.95 & 99.89 \\
\hline 102 & 2187 & 2187 & 0 & 0 & 100.00 & 100.00 & 100.00 \\
\hline 103 & 2084 & 2084 & 0 & 0 & 100.00 & 100.00 & 100.00 \\
\hline 104 & 2229 & 2222 & 7 & 10 & 99.69 & 99.55 & 99.24 \\
\hline 105 & 2572 & 2528 & 44 & 50 & 98.29 & 98.06 & 96.41 \\
\hline 106 & 2027 & 2004 & 23 & 22 & 98.87 & 98.91 & 97.80 \\
\hline 107 & 2137 & 2121 & 16 & 6 & 99.25 & 99.72 & 98.97 \\
\hline 108 & 1763 & 1739 & 24 & 18 & 98.64 & 98.98 & 97.64 \\
\hline 109 & 2532 & 2532 & 0 & 0 & 100.00 & 100.00 & 100.00 \\
\hline 111 & 2124 & 2117 & 7 & 4 & 99.67 & 99.81 & 99.48 \\
\hline 112 & 2539 & 2539 & 0 & 0 & 100.00 & 100.00 & 100.00 \\
\hline 113 & 1795 & 1795 & 0 & 0 & 100.00 & 100.00 & 100.00 \\
\hline 114 & 1879 & 1872 & 7 & 10 & 99.63 & 99.47 & 99.10 \\
\hline 115 & 1953 & 1953 & 0 & 0 & 100.00 & 100.00 & 100.00 \\
\hline 116 & 2412 & 2393 & 19 & 5 & 99.21 & 99.79 & 99.01 \\
\hline 117 & 1535 & 1534 & 1 & 1 & 99.93 & 99.93 & 99.87 \\
\hline 118 & 2278 & 2277 & 1 & 0 & 99.96 & 100.00 & 99.96 \\
\hline 119 & 1987 & 1987 & 0 & 0 & 100.00 & 100.00 & 100.00 \\
\hline 121 & 1863 & 1860 & 3 & 3 & 99.84 & 99.84 & 99.68 \\
\hline 122 & 2476 & 2476 & 0 & 0 & 100.00 & 100.00 & 100.00 \\
\hline 123 & 1518 & 1518 & 0 & 0 & 100.00 & 100.00 & 100.00 \\
\hline 124 & 1619 & 1617 & 2 & 2 & 99.88 & 99.88 & 99.75 \\
\hline 200 & 2601 & 2593 & 8 & 3 & 99.69 & 99.88 & 99.58 \\
\hline 201 & 1963 & 1962 & 1 & 1 & 99.95 & 99.95 & 99.90 \\
\hline 202 & 2136 & 2123 & 13 & 6 & 99.39 & 99.72 & 99.11 \\
\hline 203 & 2980 & 2953 & 27 & 21 & 99.09 & 99.29 & 98.40 \\
\hline 205 & 2656 & 2640 & 16 & 2 & 99.40 & 99.92 & 99.32 \\
\hline 207 & 2332 & 2018 & 314 & 328 & 86.54 & 86.02 & 75.86 \\
\hline 208 & 2955 & 2932 & 23 & 3 & 99.22 & 99.90 & 99.12 \\
\hline 209 & 3005 & 3005 & 0 & 1 & 100.00 & 99.97 & 99.97 \\
\hline 210 & 2650 & 2629 & 21 & 13 & 99.21 & 99.51 & 98.72 \\
\hline 212 & 2748 & 2748 & 0 & 0 & 100.00 & 100.00 & 100.00 \\
\hline 213 & 3251 & 3245 & 6 & 2 & 99.82 & 99.94 & 99.75 \\
\hline 214 & 2262 & 2253 & 9 & 10 & 99.60 & 99.56 & 99.16 \\
\hline 215 & 3363 & 3360 & 3 & 4 & 99.91 & 99.88 & 99.79 \\
\hline 217 & 2208 & 2193 & 15 & 10 & 99.32 & 99.55 & 98.87 \\
\hline 219 & 2154 & 2154 & 0 & 0 & 100.00 & 100.00 & 100.00 \\
\hline 220 & 2048 & 2048 & 0 & 0 & 100.00 & 100.00 & 100.00 \\
\hline 221 & 2427 & 2417 & 10 & 5 & 99.59 & 99.79 & 99.38 \\
\hline 222 & 2483 & 2480 & 3 & 3 & 99.88 & 99.88 & 99.76 \\
\hline 223 & 2605 & 2585 & 20 & 0 & 99.23 & 100.00 & 99.23 \\
\hline 228 & 2053 & 2032 & 21 & 14 & 98.98 & 99.32 & 98.31 \\
\hline 230 & 2256 & 2256 & 0 & 0 & 100.00 & 100.00 & 100.00 \\
\hline 231 & 1571 & 1571 & 0 & 0 & 100.00 & 100.00 & 100.00 \\
\hline 232 & 1780 & 1778 & 2 & 2 & 99.89 & 99.89 & 99.78 \\
\hline 233 & 3079 & 3078 & 1 & 1 & 99.97 & 99.97 & 99.94 \\
\hline 234 & 2753 & 2753 & 0 & 0 & 100.00 & 100.00 & 100.00 \\
\hline Total & 109966 & 109298 & 668 & 561 & 99.39 & 99.49 & 98.89 \\
\hline
\end{tabular}


TABLE 2: Detection results of ECG signals from QTDB.

\begin{tabular}{|c|c|c|c|c|c|c|c|}
\hline Record & $\begin{array}{l}\text { Total } \\
\text { beats }\end{array}$ & TP & $\mathrm{FN}$ & $\mathrm{FP}$ & $\begin{array}{c}\text { SEN } \\
(\%)\end{array}$ & $\begin{array}{l}+\mathrm{P} \\
(\%)\end{array}$ & $\begin{array}{c}\text { ACC } \\
(\%)\end{array}$ \\
\hline el100 & 1134 & 1134 & 0 & 0 & 100.00 & 100.00 & 100.00 \\
\hline sel102 & 088 & 1088 & 0 & 0 & 100.00 & 100.00 & 100.00 \\
\hline sel103 & 1048 & 1048 & 0 & 0 & 100.00 & 100.00 & 100.00 \\
\hline sel104 & 109 & 1109 & 0 & 1 & 100.00 & 99.91 & 99.91 \\
\hline el114 & 862 & 858 & 4 & 8 & 99.54 & 99.08 & 98.62 \\
\hline sel116 & 1185 & 1184 & 1 & 1 & 99.92 & 99.92 & 99.83 \\
\hline el117 & 766 & 766 & 0 & 0 & 100.00 & 100.00 & 100.00 \\
\hline el123 & 756 & 756 & 0 & 0 & 100.00 & 100.00 & 100.00 \\
\hline sel213 & 1642 & 1636 & 6 & 1 & 99.63 & 99.94 & 99.57 \\
\hline sel221 & 1247 & 1240 & 7 & 3 & 99.44 & 99.76 & 99.20 \\
\hline sel223 & 309 & 1307 & 2 & 2 & 99.85 & 99.85 & 99.69 \\
\hline sel230 & 1077 & 1077 & 0 & 0 & 100.00 & 100.00 & 100.00 \\
\hline sel231 & 732 & 732 & 0 & 0 & 100.00 & 100.00 & 100.00 \\
\hline sel232 & 865 & 864 & 1 & 0 & 99.88 & 100.00 & 99.88 \\
\hline sel233 & 1533 & 1507 & 26 & 1 & 98.30 & 99.93 & 98.24 \\
\hline sel301 & 1351 & 1346 & 5 & 1 & 99.63 & 99.93 & 99.56 \\
\hline sel302 & 1500 & 1498 & 2 & 1 & 99.87 & 99.93 & 99.80 \\
\hline sel306 & 1040 & 1040 & 0 & 0 & 100.00 & 100.00 & 100.00 \\
\hline sel307 & 853 & 853 & 0 & 0 & 100.00 & 100.00 & 100.00 \\
\hline sel308 & 1294 & 1285 & 9 & 5 & 99.30 & 99.61 & 98.92 \\
\hline sel310 & 2012 & 1997 & 15 & 2 & 99.25 & 99.90 & 99.16 \\
\hline sel803 & 1026 & 1026 & 0 & 0 & 100.00 & 100.00 & 100.00 \\
\hline sel808 & 903 & 902 & 1 & 1 & 99.89 & 99.89 & 99.78 \\
\hline sel811 & 704 & 704 & 0 & 0 & 100.00 & 100.00 & 100.00 \\
\hline sel820 & 1159 & 1158 & 1 & 0 & 99.91 & 100.00 & 99.91 \\
\hline sel821 & 1557 & 1556 & 1 & 1 & 99.94 & 99.94 & 99.87 \\
\hline sel840 & 1180 & 1179 & 1 & 0 & 99.92 & 100.00 & 99.92 \\
\hline sel847 & 01 & & 0 & 0 & 100.00 & 100.00 & 100.00 \\
\hline sel853 & 1113 & 1113 & 0 & 0 & 100.00 & 100.00 & 100.00 \\
\hline sel871 & 17 & & 0 & 0 & 100.00 & 100.00 & 100.00 \\
\hline sel872 & 990 & & 0 & 0 & 100.00 & 100.00 & 100.00 \\
\hline sel873 & 859 & & 1 & 1 & & 99.88 & 99.77 \\
\hline sel883 & 892 & & 1 & 2 & 99.89 & 99.78 & 99.66 \\
\hline sel891 & 1267 & 1266 & 1 & 0 & 99.92 & 100.00 & 99.92 \\
\hline sel14046 & 1260 & 1260 & 0 & 0 & 100.00 & 100.00 & 100.00 \\
\hline sel14157 & 1081 & 1081 & 0 & 0 & 100.00 & 100.00 & 100.00 \\
\hline sel14172 & 663 & 663 & 0 & 0 & 100.00 & 100.00 & 100.00 \\
\hline sel15814 & 1036 & 1035 & 1 & 0 & 99.90 & 100.00 & 99.90 \\
\hline sel16265 & 1031 & 1031 & 0 & 0 & 100.00 & 100.00 & 100.00 \\
\hline sel16272 & 851 & & 0 & 0 & 100.00 & 100.00 & 100.00 \\
\hline sel16273 & 1112 & 1111 & 1 & 0 & 99.91 & 100.00 & 99.91 \\
\hline sel16420 & 1063 & & 0 & 0 & 100.00 & 100.00 & 100.00 \\
\hline sel16483 & 1087 & 1087 & 0 & 0 & 100.00 & 100.00 & 100.00 \\
\hline sel16539 & 922 & 922 & 0 & 0 & 100.00 & 100.00 & 100.00 \\
\hline sel16773 & 1008 & 1007 & 1 & 0 & 99.90 & 100.00 & 99.90 \\
\hline sel16786 & 925 & 925 & 0 & 0 & 100.00 & 100.00 & 100.00 \\
\hline sel16795 & 761 & 761 & 0 & 0 & 100.00 & 100.00 & 100.00 \\
\hline sel17152 & 1628 & 1628 & 0 & 0 & 100.00 & 100.00 & 100.00 \\
\hline
\end{tabular}

TABle 2: Continued.

\begin{tabular}{|c|c|c|c|c|c|c|c|}
\hline Record & $\begin{array}{l}\text { Total } \\
\text { beats }\end{array}$ & TP & $\mathrm{FN}$ & $\mathrm{FP}$ & $\begin{array}{c}\text { SEN } \\
(\%)\end{array}$ & $\begin{array}{c}+\mathrm{P} \\
(\%) \\
\end{array}$ & $\begin{array}{c}\text { ACC } \\
(\%)\end{array}$ \\
\hline 117453 & 1047 & 1047 & 0 & 0 & 100.00 & 100.00 & 100.00 \\
\hline sele 0104 & 04 & 804 & 0 & 0 & 100.00 & 100.00 & 100.00 \\
\hline sele0106 & 96 & 896 & 0 & 0 & 100.00 & 100.00 & 100.00 \\
\hline sele0107 & 812 & 806 & 6 & 2 & 99.26 & 99.75 & 99.02 \\
\hline sele0110 & 872 & 870 & 2 & 9 & 99.77 & 98.98 & 98.75 \\
\hline sele0111 & 907 & 907 & 0 & 0 & 100.00 & 100.00 & 100.00 \\
\hline sele0112 & 684 & 675 & 9 & 12 & 98.68 & 98.25 & 96.98 \\
\hline sele 0114 & 699 & & 1 & 1 & 99.86 & 99.86 & 99.71 \\
\hline & 558 & & 0 & 0 & 100.00 & 100.00 & 100.00 \\
\hline & 1436 & 1431 & 5 & 0 & 99.65 & & 99.65 \\
\hline & & & 0 & 0 & 100.00 & & 100.00 \\
\hline & & & 0 & 0 & & & 100.00 \\
\hline & & & 0 & 1 & & & \\
\hline & & & 27 & 23 & & & 92.80 \\
\hline & & & 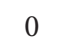 & 0 & & & 100.00 \\
\hline & & & 0 & 0 & & & 100.00 \\
\hline & & & 0 & 0 & & & 100.00 \\
\hline & & & 0 & 2 & & & 99.78 \\
\hline & 1 & & 1 & 1 & & & 99.84 \\
\hline & & & 1 & 0 & & 100.00 & 99.91 \\
\hline & & & 2 & 4 & 99.87 & 99.75 & 99.62 \\
\hline sele 0303 & 1045 & 1044 & 1 & 0 & 99.90 & 100.00 & 99.90 \\
\hline sele0405 & 1216 & 1216 & 0 & 1 & 100.00 & 99.92 & 99.92 \\
\hline sele0406 & 959 & 050 & 0 & 1 & 100.00 & 99.90 & 99.90 \\
\hline sele0409 & 1737 & 1737 & 0 & 0 & 100.00 & 100.00 & 100.00 \\
\hline sele0411 & 1202 & 1202 & 0 & 0 & 100.00 & 100.00 & 100.00 \\
\hline & 1028 & 1028 & 0 & 0 & 100.00 & 100.00 & 100.00 \\
\hline sele 0603 & & & 1 & 0 & 99.89 & 100.00 & 99.89 \\
\hline sele0604 & 1031 & 1031 & 0 & 0 & 100.00 & 100.00 & 100.00 \\
\hline sele0606 & 1442 & 1442 & 0 & 0 & 100.00 & 100.00 & 100.00 \\
\hline sele0607 & 1184 & 1184 & 0 & 0 & 100.00 & 100.00 & 100.00 \\
\hline sele0609 & 1127 & 1125 & 2 & 2 & 99.82 & 99.82 & 99.65 \\
\hline sele0612 & 751 & 751 & 0 & 0 & 100.00 & 100.00 & 100.00 \\
\hline sele0704 & 1094 & 1093 & 1 & 1 & 99.91 & 99.91 & 99.82 \\
\hline Total & 86995 & 86848 & 147 & 91 & 99.83 & 99.90 & 99.73 \\
\hline
\end{tabular}

However, for records 107, 109, 113, and 116 in MITDB, our method consumes the same and even more time than the Pan-Tompkins method. The disadvantage of our method is plotted in Figure 9 versus the Pan-Tompkins method in terms of time complexity. In each subfigure, the abscissa represents the quantitative increment of samples. The basic number of samples is 720 ; it is multiplied by the number shown in the abscissa. In the top row, the ordinate is the multiple increments of time consumed by processing the multiplied samples shown in the abscissa. In the bottom row, the ordinates are the increment of time multiples calculated from the ordinates in the top row. Intuitively, the four records have high increments of time consumption as the amount of data 
TABLE 3: Comparison of R-peak detection with other algorithms.

\begin{tabular}{|c|c|c|c|c|c|c|c|c|}
\hline & Dataset & Beats & $\mathrm{TP}$ & FN & FP & SEN (\%) & $+\mathrm{P}(\%)$ & ACC (\%) \\
\hline Zidelmal et al. [12] & MITDB & 109494 & 109101 & 393 & 193 & 99.64 & 99.82 & 99.47 \\
\hline Pan and Tompkins [15] & MITDB & 116137 & 115860 & 277 & 507 & 99.76 & 99.56 & 99.33 \\
\hline Jung and Lee [21] & MITDB & 109541 & 108960 & 581 & 579 & 99.47 & 99.47 & 98.94 \\
\hline Chiarugi et al. [38] & MITDB & 109494 & 109288 & 266 & 210 & 99.76 & 99.81 & 99.57 \\
\hline Arzeno et al. [39] & MITDB & 109517 & 109099 & 354 & 405 & 99.68 & 99.63 & 99.31 \\
\hline Elgendi [40] & MITDB & 109985 & 109738 & 247 & 124 & 99.78 & 99.87 & 99.66 \\
\hline Christov [41] & MITDB & 110050 & 109615 & 240 & 239 & 99.74 & 99.65 & 99.56 \\
\hline Chouakri et al. [42] & MITDB & 110934 & 109488 & 1446 & 3068 & 98.68 & 97.24 & 96.03 \\
\hline Rodríguez et al. [43] & MITDB & 44715 & 42518 & 879 & 142 & 96.28 & 99.71 & 97.65 \\
\hline Yeh and Wang [44] & MITDB & 116137 & 115971 & 166 & 58 & 99.86 & 99.95 & 99.81 \\
\hline \multirow{2}{*}{ The proposed } & MITDB & 109966 & 109298 & 668 & 561 & 99.39 & 99.49 & 98.89 \\
\hline & QTDB & 86995 & 86848 & 147 & 91 & 99.83 & 99.90 & 99.73 \\
\hline
\end{tabular}

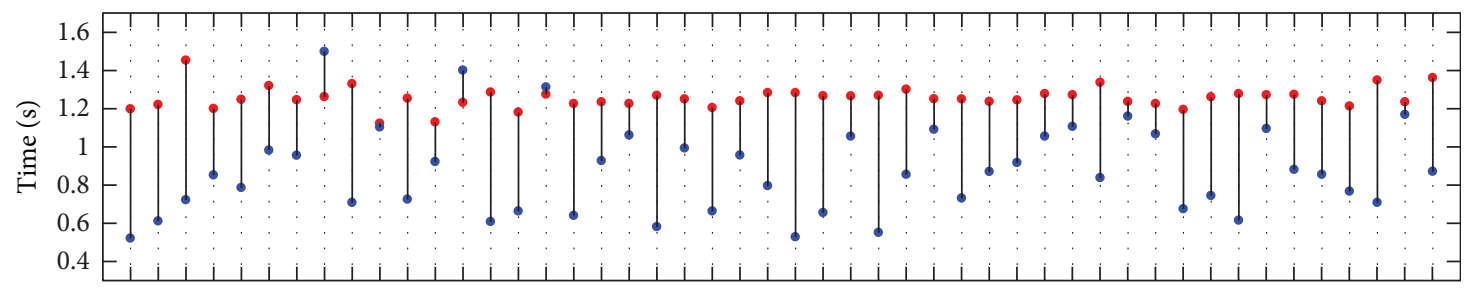

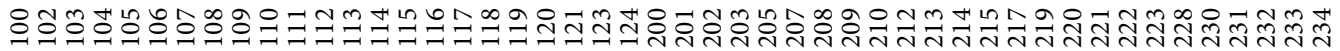

Record number

- Pan-Tompkins method

- Proposed

(a) Time of processing one record (MITDB)

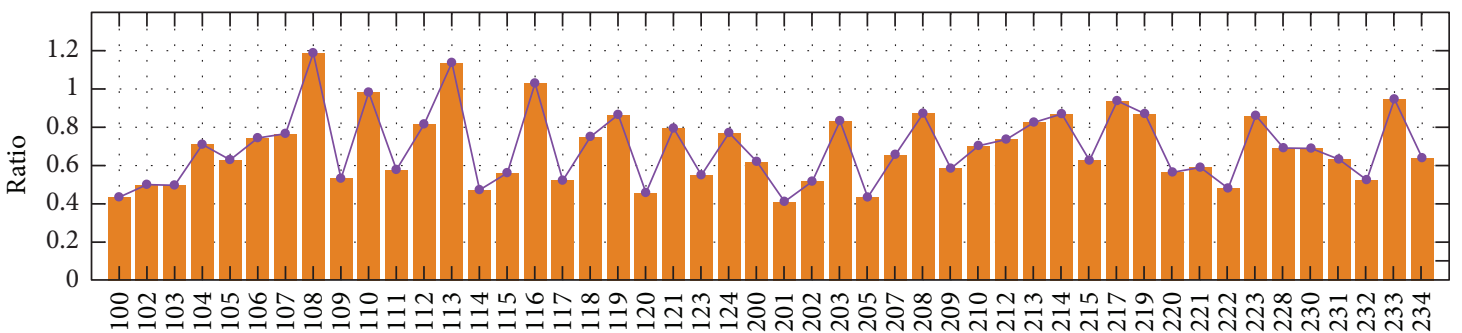

Record number

(b) Time ratio of processing one record (MITDB)

FIgURE 6: (a) Time consumption of processing the 48 records in MITDB and (b) time consumption ratio of the proposed method over the Pan-Tompkins method.

increases, especially when the data quantity is less than 500 times of the basic number 720 . One important reason is that the four records contain numerous large $\mathrm{T}$ waves or $\mathrm{P}$ waves to be compared by time interval threshold or large negative amplitudes to be estimated for mirroring. Time interval comparison is more frequent for these waveforms, leading to higher time complexity than that of the Pan-Tompkins method. But on the other hand, the curves prove that our algorithm is not so sensitive to data increase. The increments of time multiple fluctuate in small ranges, basically remaining unchanged. The multiples of time consumption have linear relationships with the increments of sample amount.

4.3. $K_{\text {amp }}$ and $K_{\text {time }}$. Amplitude threshold takes a significant role in truncating burst points along the baseline; time interval threshold is a critical measure to further distinguish false R-peaks. According to (3), the thresholds depend significantly on $K_{\text {amp }}$ and $K_{\text {time }}$. To determine optimal coefficients and validate the feasibility of adaptive thresholding, different a_t and ti_t values are tested using the 48 ECG records in 


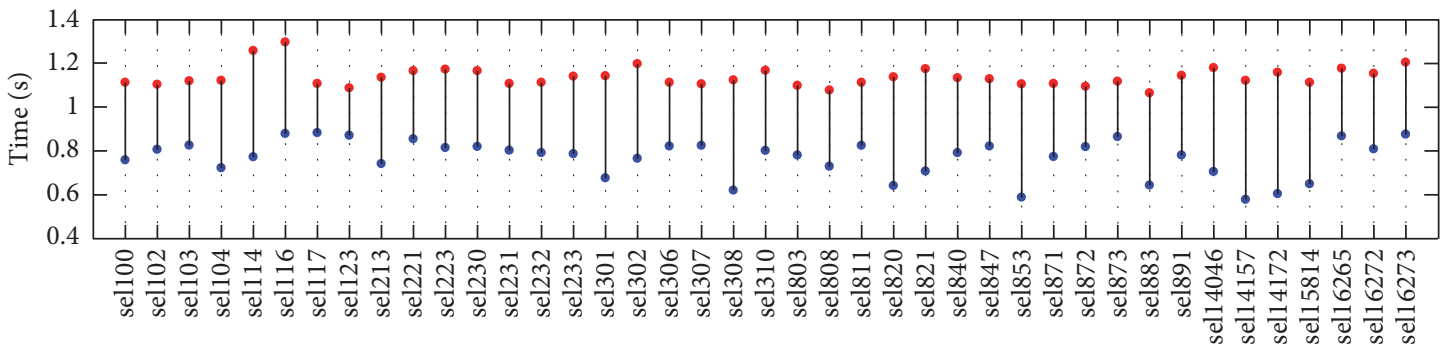

Record number

- Pan-Tompkins method

- Proposed

(a) Time of processing one record (first 41 records from QTDB)

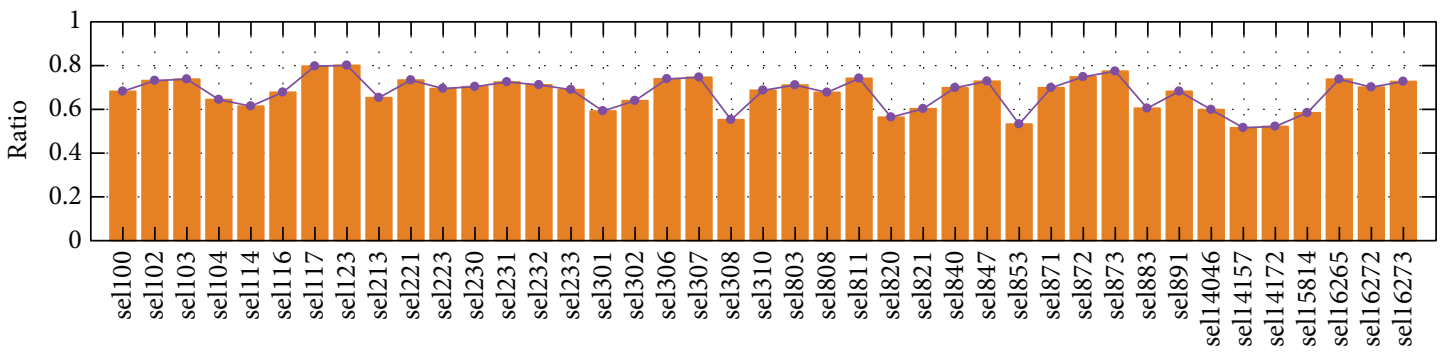

Record number

(b) Time ratio of processing one record (first 41 records from QTDB)

FIGURE 7: (a) Time consumption of processing the first 41 records in QTDB and (b) time consumption ratio of the proposed method over the Pan-Tompkins method.

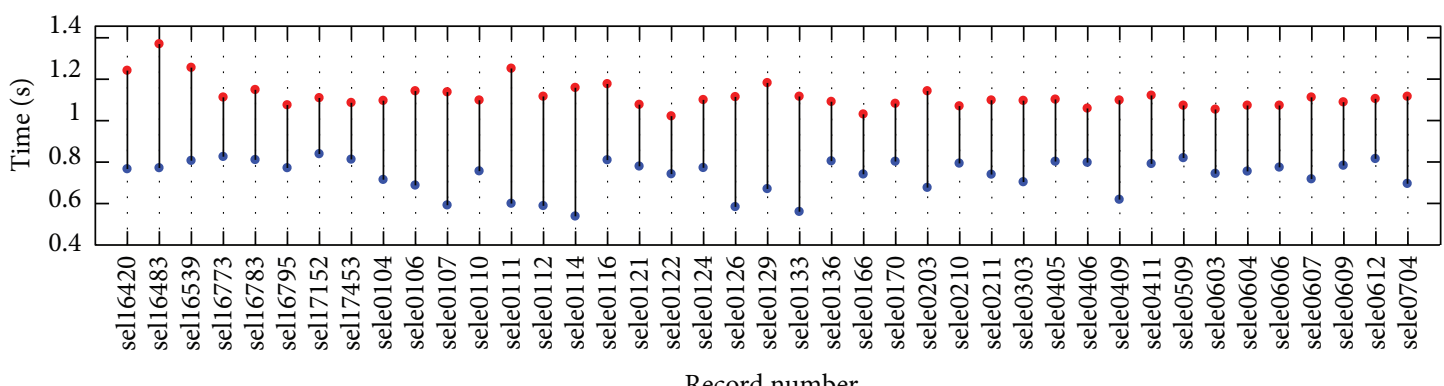

- Pan-Tompkins method

- Proposed

(a) Time of processing one record (last 41 records from QTDB)

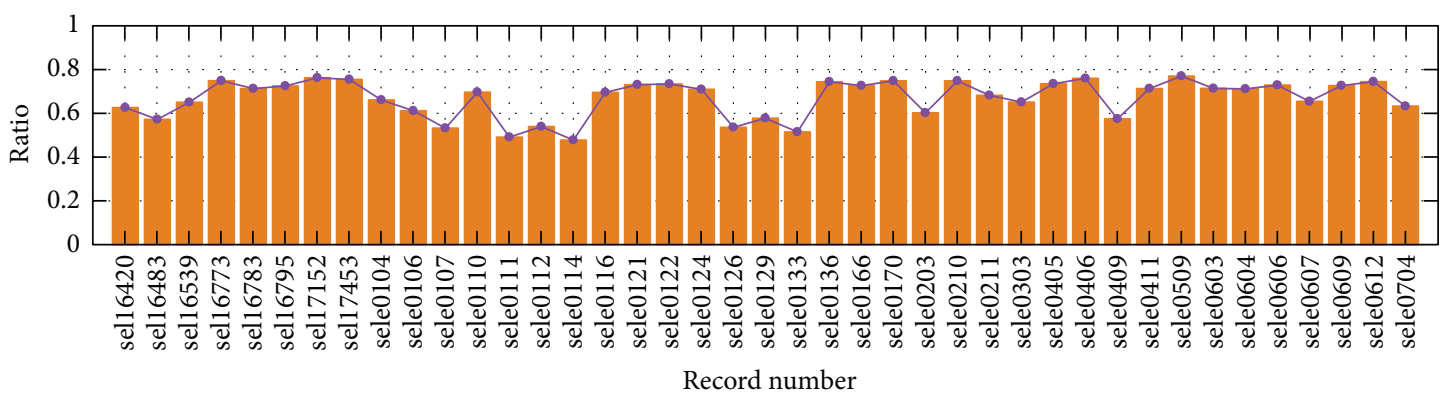

(b) Time ratio of processing one record (last 41 records from QTDB)

FIGURE 8: (a) Time consumption of processing the last 41 records in QTDB and (b) time consumption ratio of the proposed method over the Pan-Tompkins method. 

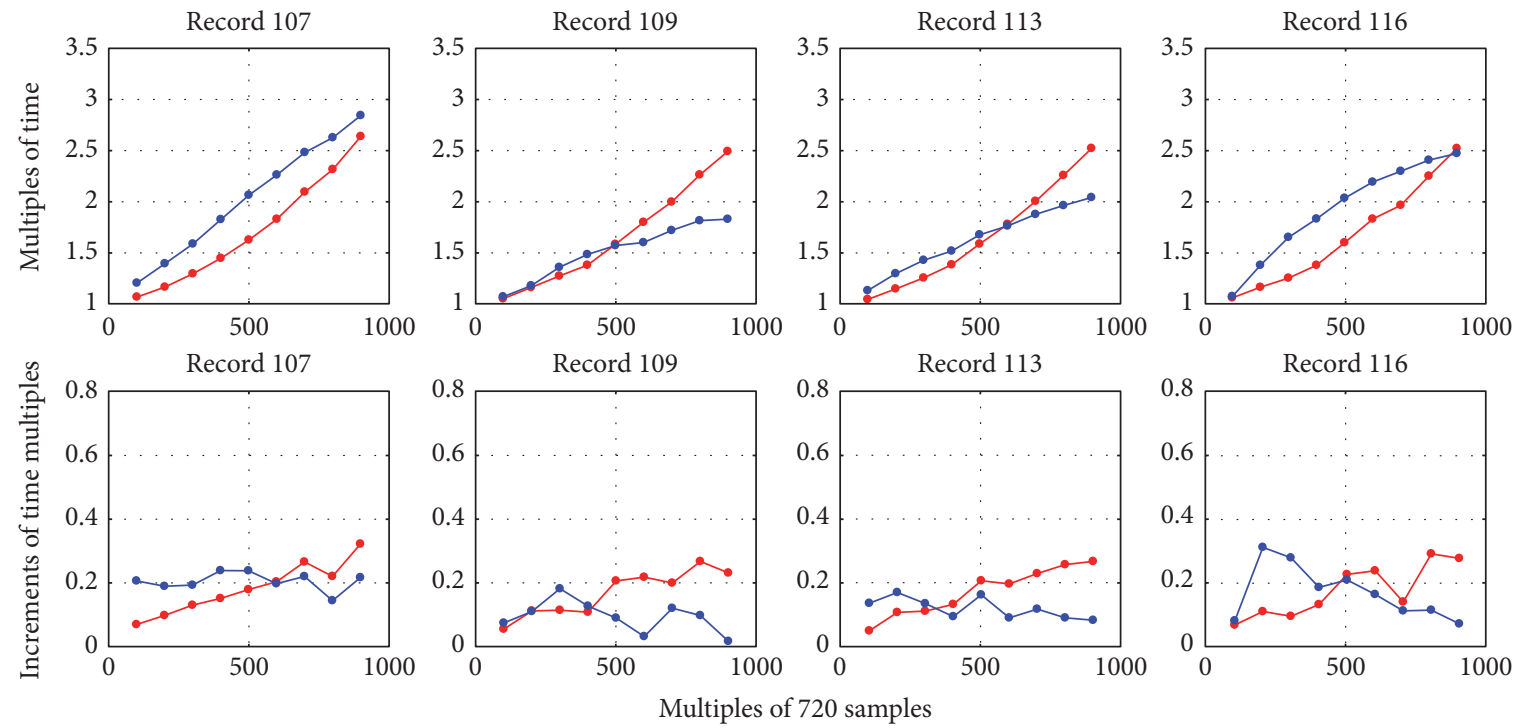

_ Pan-Tompkins method

— Proposed

FIGURE 9: Multiples of time consumption versus multiples of samples.

TABle 4: Comparison of different threshold coefficients.

\begin{tabular}{|c|c|c|c|c|c|c|c|c|c|c|}
\hline & $K_{\text {time }}$ & 0.10 & 0.15 & 0.20 & 0.25 & $\begin{array}{c}K_{\mathrm{amp}} \\
0.30\end{array}$ & 0.35 & 0.40 & 0.45 & 0.50 \\
\hline SEN (\%) & & 98.65 & 98.93 & 98.68 & 97.87 & 96.45 & 93.87 & 90.78 & 87.54 & 84.16 \\
\hline +P (\%) & 0.36 & 80.94 & 87.29 & 90.23 & 92.38 & 93.69 & 94.55 & 96.56 & 97.89 & 98.67 \\
\hline $\operatorname{ACC}(\%)$ & & 80.06 & 86.47 & 89.16 & 90.56 & 90.57 & 89.05 & 87.94 & 85.92 & 83.22 \\
\hline SEN (\%) & & 96.42 & 97.59 & 98.05 & 97.61 & 96.31 & 93.79 & 90.72 & 87.51 & 84.14 \\
\hline +P (\%) & 0.39 & 85.94 & 91.24 & 93.64 & 94.99 & 95.58 & 96.37 & 98.08 & 99.04 & 99.39 \\
\hline ACC (\%) & & 83.28 & 89.24 & 91.93 & 92.83 & 92.20 & 90.59 & 89.14 & 86.78 & 83.71 \\
\hline SEN (\%) & & 95.70 & 97.20 & 97.80 & 97.43 & 96.18 & 93.68 & 90.64 & 87.44 & 84.09 \\
\hline +P (\%) & 0.42 & 89.98 & 94.56 & 96.42 & 97.40 & 97.79 & 98.27 & 98.98 & 99.38 & 99.56 \\
\hline ACC (\%) & & 86.48 & 92.05 & 94.38 & 94.96 & 94.13 & 92.16 & 89.80 & 86.97 & 83.78 \\
\hline SEN (\%) & & 94.57 & 96.47 & 97.17 & 99.39 & 95.73 & 93.36 & 90.42 & 87.28 & 83.94 \\
\hline +P (\%) & 0.45 & 92.51 & 96.73 & 98.28 & 99.49 & 99.09 & 99.26 & 99.42 & 99.54 & 99.63 \\
\hline ACC (\%) & & 90.85 & 96.42 & 98.54 & 98.89 & 97.90 & 95.72 & 92.94 & 89.93 & 86.68 \\
\hline SEN (\%) & & 93.84 & 95.87 & 96.63 & 96.38 & 95.26 & 92.90 & 89.97 & 86.86 & 83.57 \\
\hline +P (\%) & 0.48 & 93.54 & 97.20 & 98.59 & 99.07 & 99.26 & 99.38 & 99.48 & 99.56 & 99.64 \\
\hline ACC (\%) & & 88.13 & 93.29 & 95.32 & 95.52 & 94.58 & 92.37 & 89.55 & 86.53 & 83.31 \\
\hline SEN (\%) & & 93.06 & 95.28 & 96.08 & 95.83 & 94.69 & 92.30 & 89.35 & 86.24 & 82.98 \\
\hline +P (\%) & 0.51 & 94.04 & 97.45 & 98.68 & 99.10 & 99.28 & 99.39 & 99.49 & 99.56 & 99.64 \\
\hline ACC (\%) & & 87.87 & 92.96 & 94.86 & 95.01 & 94.04 & 91.78 & 88.94 & 85.92 & 82.73 \\
\hline SEN (\%) & & 92.10 & 94.59 & 95.41 & 95.15 & 94.02 & 91.66 & 88.73 & 85.66 & 82.43 \\
\hline +P (\%) & 0.54 & 94.26 & 97.63 & 98.73 & 99.12 & 99.30 & 99.41 & 99.50 & 99.57 & 99.65 \\
\hline ACC (\%) & & 87.21 & 92.47 & 94.25 & 94.36 & 93.40 & 91.16 & 88.34 & 85.35 & 82.19 \\
\hline SEN (\%) & & 90.90 & 93.54 & 94.41 & 94.14 & 93.03 & 90.73 & 87.86 & 84.85 & 81.65 \\
\hline +P (\%) & 0.57 & 94.31 & 97.68 & 98.76 & 99.15 & 99.31 & 99.42 & 99.51 & 99.57 & 99.65 \\
\hline $\operatorname{ACC}(\%)$ & & 86.17 & 91.51 & 93.30 & 93.38 & 92.44 & 90.25 & 87.48 & 84.54 & 81.42 \\
\hline SEN (\%) & & 89.10 & 91.87 & 92.80 & 92.60 & 91.58 & 89.46 & 86.85 & 83.99 & 80.90 \\
\hline$+\mathrm{P}(\%)$ & 0.6 & 94.28 & 97.67 & 98.76 & 99.16 & 99.32 & 99.43 & 99.52 & 99.58 & 99.65 \\
\hline $\operatorname{ACC}(\%)$ & & 84.53 & 89.90 & 91.73 & 91.88 & 91.01 & 89.00 & 86.48 & 83.69 & 80.68 \\
\hline
\end{tabular}




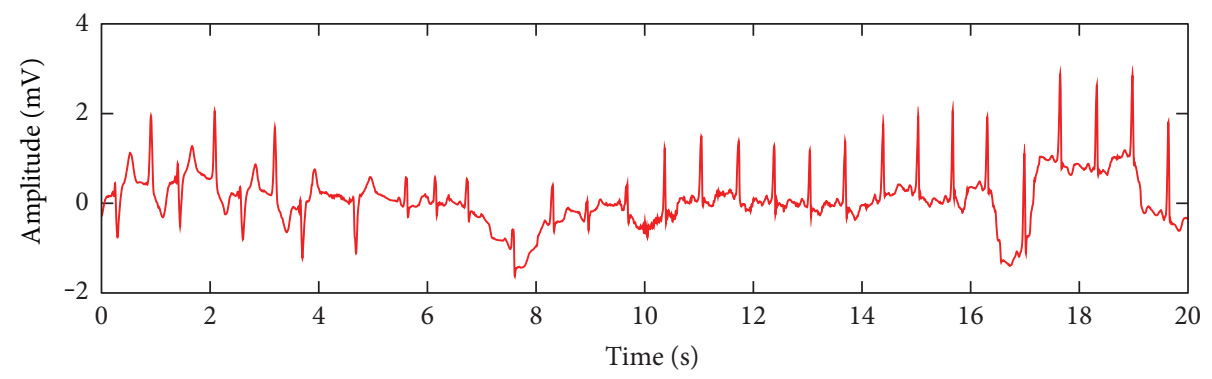

(a) Record 223 with different interface

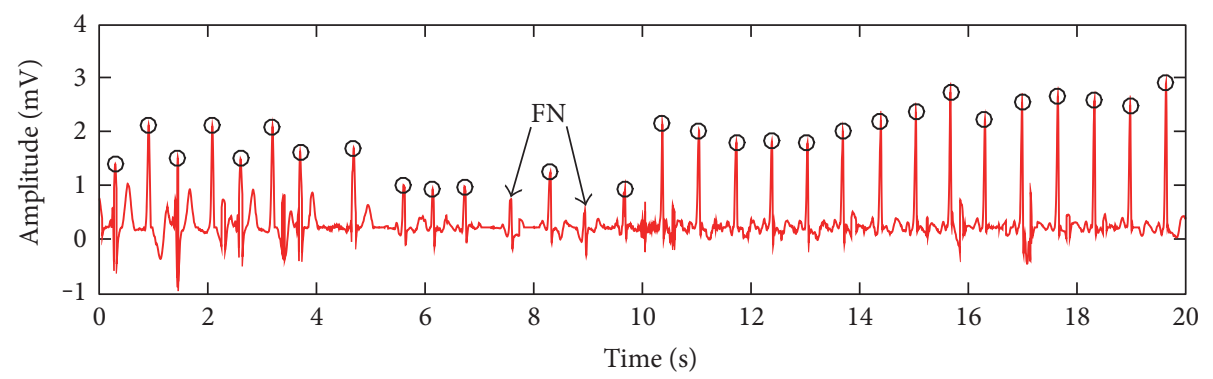

(b) Missing detection of small R-peaks on record 223

Figure 10: Missing detection due to the small amplitude of R-peaks.

MITDB. RR intervals of the records range from $0.54 \mathrm{~s}$ to $1.19 \mathrm{~s}$; an average of $0.82 \mathrm{~s}$ is adopted to cope with different heart rates. The validation results are summarized in Table 4.

It can be seen that the optimal a_t is generally $K_{\text {amp }}=0.2 \sim 0.3$ times the $\mathrm{MAX}_{\text {seg_amp}}$, with $K_{\text {amp }}=0.25$ reaching the maximum $\mathrm{SEN},+\mathrm{P}$ and $\mathrm{ACC}$ as illustrated in Table 1. On one hand, large a_t is beneficial for the algorithm to cut off points located near the baseline, but small true Rpeaks may be missed if a_t is too large. As shown in the table, when $K_{\mathrm{amp}}$ is larger than 0.40 , most of the false R-peaks are filtered as well as true ones, resulting in high FN but low $\mathrm{FP}$ and correspondingly low SEN but high $+\mathrm{P}$. On the other, smaller a_t generates fewer omitted R-peaks, but this will increase the computational cost in time interval thresholding. The worst situation is that all the potential R-peaks are compared, as revealed in the table when $K_{a m p}$ is smaller than 0.20. In this case, most of the true R-peaks are included in local maximums as well as false ones, resulting in low FN but high FP and correspondingly high SEN but low $+P$. To coordinate with different heart rates, $K_{\mathrm{amp}}$ is recommended to be selected from interval $[0.2,0.3]$ and $K_{\text {time }}$ from [0.42, 0.48]. After $K_{\mathrm{amp}}$ and $K_{\text {time }}$ are determined, a_t and ti_t can be automatically updated by the shift of new coming signal.

\section{Discussion}

The proposed method has two advantages. One is from the time efficiency as indicated in Section 4.2. The main difference between our method and the Pan-Tompkins method is that the latter calculates more measures for Rpeak recognition. It includes a search back operation after a complete detection circulation, thus resulting in a high computational complexity. Our method exclusively uses the thresholding method, and it does not require any search back operation. Besides, the amplitude threshold can also contribute to the calculation efficiency since it excludes most distractors and significantly reduces the amount of threshold comparisons.

Another advantage is from that there is no time length limitation for thresholding. As described in Section 2.4, the length of new coming segment is flexible, and the thresholding procedure can operate not only for a single heartbeat but also for a complete ECG record. With adaptive $K_{\mathrm{amp}}$ and $K_{\text {time }}$, our method is suitable for different lengths of ECG signal and it requires no prelearning procedure.

From Table 1, we can see that about half of the failed beats (314 FN and $328 \mathrm{FP}$ ) are from record 207. This record consists of numerous distorted heartbeats that are extremely difficult to be recognized even by a cardiologist. However, few literatures reported the results on this specific record. It is also unclear if record 207 is excluded in the evaluations to achieve a high score. This record is also the main interference that significantly reduces the detection accuracy of our method.

Apart from record 207, there are still some missing and false recognitions on some other records. The main methodological defect of the algorithm is that amplitude threshold may fail to detect small R-peaks mixed in large ones (records $105,106,108$, and 228). The a_t is selected based on the local maximums; if a segment contains numerous large R-peaks, a_t will be larger than small $\mathrm{R}$-peaks. This weights against the identification for small R-peaks because they are prone to be partitioned below the a_t, as illustrated in Figure 10. The signal contains baseline drift, large $\mathrm{T}$ waves, and large negative amplitude R-peaks. Although most of the R-peaks are recognized, there are two $\mathrm{FN}$ detections for small Rpeaks. If an R-peak is missed, ti_t would probably take 
the following maximum as the candidate R-peak, which actually is a distractor.

\section{Conclusions}

In this study, an adaptive and time-efficient methodology has been developed for automatic ECG R-peak detection. It is an adaptive method integrating WMRA, signal mirroring, local maximum detection, and amplitude and time interval thresholding. The accuracy performances were tested by using ECG records from MITDB and QTDB. Experimental results indicate that the proposed algorithm achieves average SEN, $+\mathrm{P}$, and ACC of $99.39 \%$, 99.49\%, and $98.89 \%$ for MITDB, and $99.83 \%, 99.90 \%$, and $99.73 \%$ for QTDB, respectively. In addition, time consumption and time complexity of the algorithm are computed to prove its time efficiency. By processing one ECG record, the average time cost is $0.872 \mathrm{~s}$ for MITDB and $0.763 \mathrm{~s}$ for QTDB, achieving 30.6\% and $32.9 \%$, respectively, of time reduction compared to the Pan-Tompkins method. Experiments on time complexity demonstrate that the proposed method is provided with linear time complexity; both our method and the PanTompkins method are less sensitive to data increase.

\section{Conflicts of Interest}

The authors declare that they have no conflicts of interest.

\section{Acknowledgments}

This study is supported by the National Natural Science Foundation of China (61571113). This study is also supported by the Natural Science Foundation of Jiangsu Province of China (BK20160697), the International S\&T Cooperation Program of China (2015DFA10490), and the China Scholarship Council (CSC).

\section{References}

[1] J. R. Windmiller and J. Wang, "Wearable electrochemical sensors and biosensors: a review," Electroanalysis, vol. 25, no. 1, pp. 29-46, 2013.

[2] L. Atallah, A. Serteyn, M. Meftah et al., "Unobtrusive ECG monitoring in the NICU using a capacitive sensing array," Physiological Measurement, vol. 35, no. 5, pp. 895-913, 2014.

[3] B. Taji, V. G. Shirmohammadi, and I. Batkin, "Impact of skin-electrode interface on electrocardiogram measurements using conductive textile electrodes," IEEE Transactions on Instrumentation and Measurement, vol. 63, no. 6, pp. 14121422, 2014.

[4] P. Augustyniak, "Wearable wireless heart rate monitor for continuous long-term variability studies," Journal of Electrocardiology, vol. 44, no. 2, pp. 195-200, 2011.

[5] Y. M. Chi, T. P. Jung, and G. Cauwenberghs, "Dry-contact and noncontact biopotential electrodes: methodological review," IEEE Reviews in Biomedical Engineering, vol. 3, pp. 106-119, 2010.

[6] H. F. Posada-Quintero, B. A. Reyes, K. Burnham, J. Pennace, and K. H. Chon, "Low impedance carbon adhesive electrodes with long shelf life," Annals of Biomedical Engineering, vol. 43, no. 10, pp. 2374-2382, 2015.

[7] E. M. Fong and W. Y. Chung, "A hygroscopic sensor electrode for fast stabilized non-contact ECG signal acquisition," Sensors, vol. 15, no. 8, pp. 19237-19250, 2015.

[8] H. Y. Lin, S.-Y. Liang, Y.-L. Ho, Y.-H. Lin, and H.-P. Ma, "Discrete-wavelet-transform-based noise removal and feature extraction for ECG signals," IRBM, vol. 35, no. 6, pp. 351361, 2014.

[9] W. Tylman, T. Waszyrowski, A. Napieralski et al., "Real-time prediction of acute cardiovascular events using hardwareimplemented Bayesian networks," Computers in Biology and Medicine, vol. 69, pp. 245-253, 2016.

[10] R. Gutierrez-Rivas, J. J. García, W. P. Marnane, and Á. Hernández, "Novel real-time low-complexity QRS complex detector based on adaptive thresholding," IEEE Sensors Journal, vol. 15, no. 10, pp. 6036-6043, 2015.

[11] H. Naseri and M. R. Homaeinezhad, "Electrocardiogram signal quality assessment using an artificially reconstructed target lead," Computer Methods in Biomechanics and Biomedical Engineering, vol. 18, no. 10, pp. 1126-1141, 2015.

[12] Z. Zidelmal, A. Amirou, M. Adnane, and A. Belouchrani, "QRS detection based on wavelet coefficients," Computer Methods and Programs in Biomedicine, vol. 107, no. 3, pp. 490-496, 2012.

[13] R. Benali, F. B. Reguig, and Z. H. Slimane, "Automatic classification of heartbeats using wavelet neural network," Journal of Medical Systems, vol. 36, no. 2, pp. 883-892, 2012.

[14] M. Elgendi, B. Eskofier, S. Dokos, and D. Abbott, "Revisiting QRS detection methodologies for portable, wearable, batteryoperated, and wireless ECG systems," PloS One, vol. 9, no. 1, article e84018, 2014.

[15] J. Pan and W. J. Tompkins, "A real time QRS detection algorithm," IEEE Transactions on Biomedical Engineering, vol. 32, no. 3, pp. 230-236, 1985.

[16] B. Abibullaev and H. D. Seo, "A new QRS detection method using wavelets and artificial neural networks," Journal of Medical Systems, vol. 35, no. 4, pp. 683-691, 2011.

[17] F. Khelifi and J. M. Jiang, "K-NN regression to improve statistical feature extraction for texture retrieval," IEEE Transactions on Image Processing, vol. 20, no. 1, pp. 293-298, 2011.

[18] D. Clifton, P. S. Addison, M. K. Stiles et al., "Using wavelet transform reassignment techniques for ECG characterization," 30th Annual Meeting on Computers in Cardiology, vol. 30, pp. 581-584, 2003.

[19] F. Zhang and Y. Lian, "QRS detection based on multi-scale mathematical morphology for wearable ECG devices in body area networks," IEEE Transactions on Biomedical Circuits and Systems, vol. 3, no. 4, pp. 220-228, 2009.

[20] C. So-In, C. Phaudphut, and K. Rujirakul, "Real-time ECG noise reduction with QRS complex detection for mobile health services," Arabian Journal for Science and Engineering, vol. 40, no. 9, pp. 2503-2514, 2015.

[21] W. H. Jung and S. G. Lee, "An R-peak detection method that uses an SVD filter and a search back system," Computer Methods and Programs in Biomedicine, vol. 108, no. 3, pp. 1121-1132, 2012.

[22] S. Banerjee and M. Mitra, “A cross wavelet transform based approach for ECG feature extraction and classification without denoising," in Proceedings of The 2014 International 
Conference on Control, Instrumentation, Energy and Communication (CIEC), pp. 162-165, Calcutta, India, 2014.

[23] J. Li, X. Li, B. Yang, and X. Sun, "Segmentation-based image copy-move forgery detection scheme," IEEE Transactions on Information Forensics and Security, vol. 10, no. 3, pp. 507518, 2015.

[24] E. B. Mazomenos, D. Biswas, A. Acharyya et al., "A lowcomplexity ECG feature extraction algorithm for mobile healthcare applications," IEEE Journal of Biomedical and Health Informatics, vol. 17, no. 2, pp. 459-469, 2013.

[25] http://physionet.org/cgi-bin/atm/ATM.

[26] S. Pal and M. Mitra, "Empirical mode decomposition based ECG enhancement and QRS detection," Computers in Biology and Medicine, vol. 42, no. 1, pp. 89-92, 2012.

[27] J. H. Kim, "Discrete wavelet transform-based feature extraction of experimental voltage signal for li-ion cell consistency," IEEE Transactions on Vehicular Technology, vol. 65, no. 3, pp. 1150-1161, 2016.

[28] M. A. Kabir and C. Shahnaz, "Denoising of ECG signals based on noise reduction algorithms in EMD and wavelet domains," Biomedical Signal Processing and Control, vol. 7, no. 5, pp. 481-489, 2012.

[29] D. L. Donoho, "De-noising by soft thresholding," IEEE Transactions on Information Theory, vol. 41, no. 3, pp. 613627, 1995.

[30] M. A. Awal, S. S. Mostafa, M. Ahmad, and M. A. Rashid, “An adaptive level dependent wavelet thresholding for ECG denoising," Biocybernetics and Biomedical Engineering, vol. 34, no. 4, pp. 238-249, 2014.

[31] S. Satheeskumaran and M. Sabrigiriraj, "A new LMS based noise removal and DWT based R-peak detection in ECG signal for biotelemetry applications," National Academy Science Letters, vol. 37, no. 4, pp. 341-349, 2014.

[32] M. Merah, T. A. Abdelmalik, and B. H. Larbi, "R-peaks detection based on stationary wavelet transform," Computer Methods and Programs in Biomedicine, vol. 121, no. 3, pp. 149-160, 2015.

[33] S. D. Xie and Y. X. Wang, "Construction of tree network with limited delivery latency in homogeneous wireless sensor networks," Wireless Personal Communications, vol. 78, no. 1, pp. 231-246, 2014.

[34] D. Komorowski, S. Pietraszek, E. Tkacz, and I. Provaznik, “The extraction of the new components from electrogastrogram (EGG) using both adaptive filtering and electrocardiographic (ECG) derived respiration signal," Biomedical Engineering Online, vol. 14, 2015.

[35] A. L. Goldberger, L. A. Amaral, L. Glass et al., "PhysioBank, PhysioToolkit, and PhysioNet: components of a new research resource for complex physiologic signals," Circulation, vol. 101, no. 23, pp. e215-e220, 2000.

[36] G. B. Moody and G. R. Mark, "The impact of the MIT-BIH arrhythmia database," IEEE Engineering in Medicine and Biology Magazine, vol. 20, no. 3, pp. 45-50, 2001.

[37] P. Laguna, R. G. Mark, A. Goldberg, and G. B. Moody, "A database for evaluation of algorithms for measurement of QT and other waveform intervals in the ECG," Computers in Cardiology, vol. 24, pp. 673-676, 1997.

[38] F. Chiarugi, V. Sakkalis, D. Emmanouilidou, T. Krontiris, M. Varanini, and I. Tollis, "Adaptive threshold QRS detector with best channel selection based on a noise rating system," Computers in Cardiology, vol. 34, pp. 157-160, 2007.
[39] N. M. Arzeno, Z. D. Deng, and C. S. Poon, "Analysis of first-derivative based QRS detection algorithms," IEEE Transactions on Biomedical Engineering, vol. 55, no. 2, pp. 478-484, 2008.

[40] M. Elgendi, "Fast QRS detection with an optimized knowledge-based method: evaluation on 11 standard ECG databases," PLoS One, vol. 8, no. 9, article e73557, 2013.

[41] I. I. Christov, "Real time electrocardiogram QRS detection using combined adaptive threshold," Biomedical Engineering Online, vol. 3, no. 28, pp. 1-9, 2004.

[42] S. A. Chouakri, F. B. Reguig, and A. Taleb-Ahmed, "QRS complex detection based on multi wavelet packet decomposition," Applied Mathematics and Computation, vol. 217, no. 23, pp. 9508-9525, 2011.

[43] R. Rodríguez, A. Mexicano, J. Bila, S. Cervantes, and R. Ponce, "Feature extraction of electrocardiogram signals by applying adaptive threshold and principal component analysis," Journal of Applied Research and Technology, vol. 13, no. 2, pp. 261269, 2015.

[44] Y. C. Yeh and W. J. Wang, "QRS complexes detection for ECG signal: the difference operation method," Computer Methods and Programs in Biomedicine, vol. 91, no. 3, pp. 245-254, 2008. 


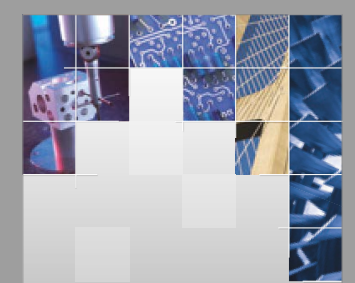

\section{Enfincering}
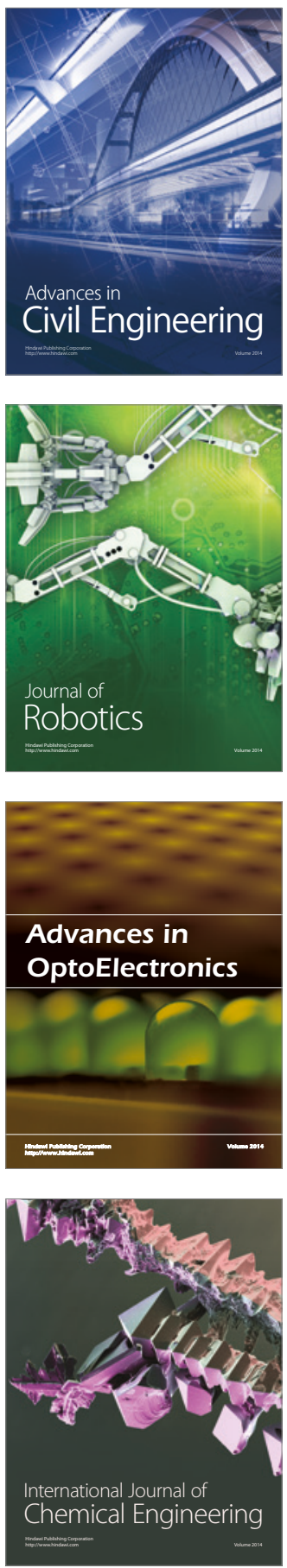

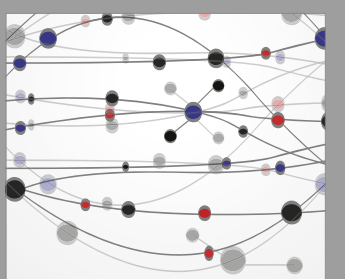

The Scientific World Journal

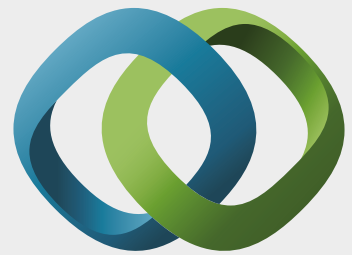

\section{Hindawi}

Submit your manuscripts at

https://www.hindawi.com
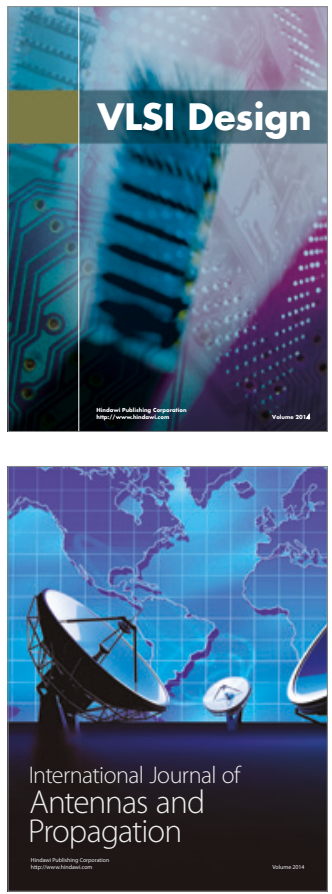

\section{Rotating}

Machinery
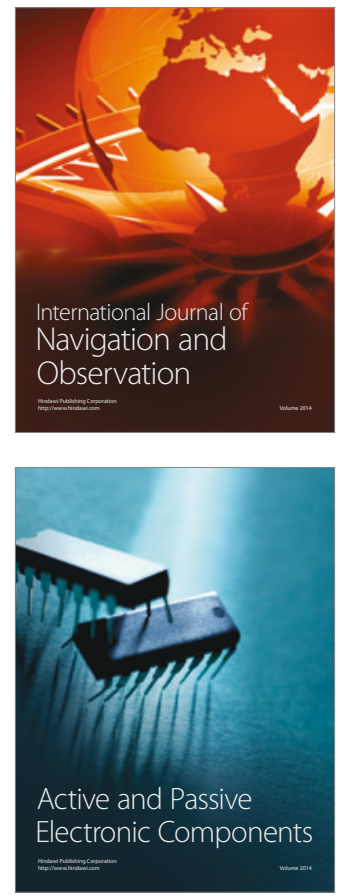
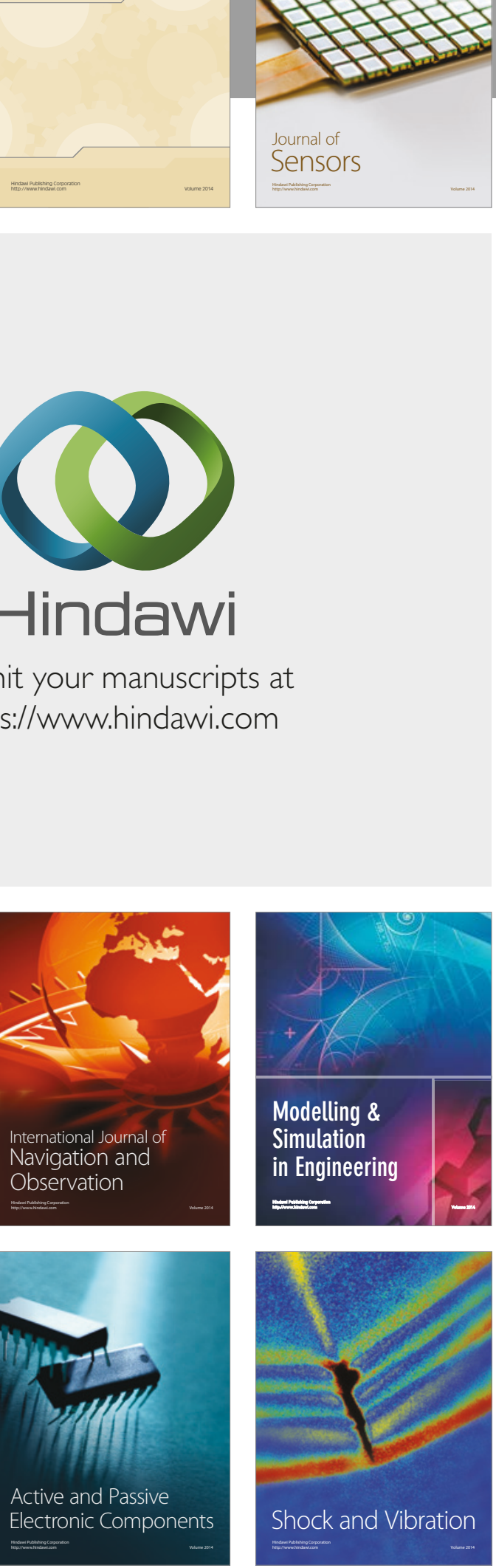
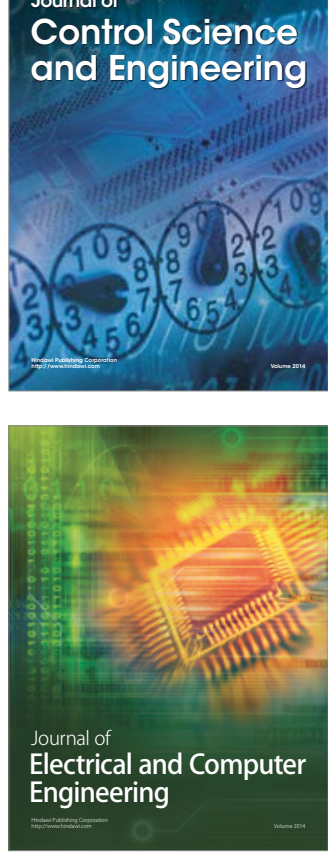

Distributed

Journal of

Control Science

and Engineering
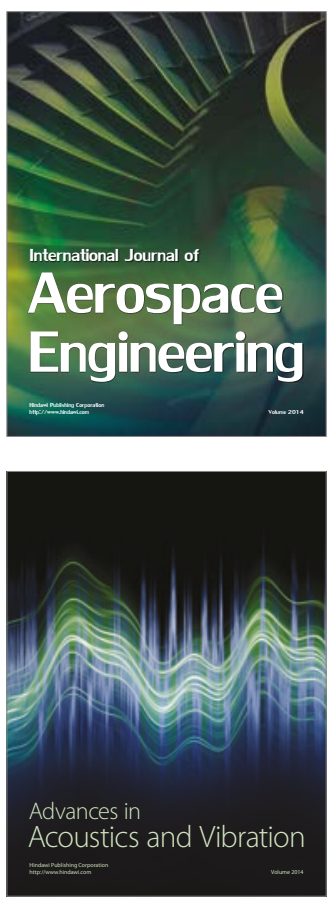

Sensor Networks 